\title{
Non-alcoholic fatty liver disease in non-obese children
}

\author{
Katarzyna Zdanowicz ${ }^{1}$, MD, Irena Białokoz-Kalinowska², PhD, MD, Dariusz M Lebensztejn ${ }^{1}, \mathrm{PhD}, \mathrm{MD}$ \\ ${ }^{1}$ Department of Pediatrics, Gastroenterology, Hepatology, Nutrition and Allergology, Medical University of Bialystok, Bialystok, Poland \\ ${ }^{2}$ Medical Institute, Lomza State University of Applied Sciences, Lomza, Poland \\ * Corresponding author: kazdanowicz@gmail.com
}

Hong Kong Med J 2020;26:459-62

https://doi.org/10.12809/hkmj198361

\section{Introduction}

Non-alcoholic fatty liver disease (NAFLD) is a broad spectrum of liver diseases, morphologically characterised by fat accumulation in $>5 \%$ of hepatocyte in the absence of other cause of steatosis. ${ }^{1}$ Less-common factors of hepatic steatosis include metabolic disorders (eg, cystic fibrosis, Wilson's disease, alpha-1 antitrypsin deficiency, galactosaemia, hereditary fructose intolerance), general or systemic disorders (eg, celiac disease, hepatitis C, obstructive sleep apnoea, type 1 diabetes), starvation, malnutrition, total parenteral nutrition, and drug toxicity. ${ }^{2}$

In recent decades, the increase in the prevalence of NAFLD is related to the global obesity epidemic and is considered to be a hepatic manifestation of the metabolic syndrome. ${ }^{2}$ Exposure to certain factors such as maternal overnutrition, gestational diabetes mellitus, Caesarean section, intrauterine growth retardation, and antibiotic use during pregnancy and infancy may be associated with an increased risk of NAFLD in children. In contrast, breastfeeding is a potential protective factor. ${ }^{3}$

Recent data have shown that NAFLD can occur in patients with a normal body mass index (BMI). Metabolically obese, lean NAFLD patients are non-obese subjects (BMI <95th percentile of age, overweight is not an exclusion criterion) with or without coexisting increased waist circumference and visceral adipose tissue. ${ }^{2}$

\section{Epidemiology}

The incidence of lean NAFLD in adolescents varies widely, ranging from $8 \%$ in the US4 to $16 \%$ in the Asia-Pacific region., ${ }^{4,5}$ Large discrepancies in the occurrence rates may be related to different inclusion criteria and cut-off points for non-obese NAFLD. ${ }^{6,7}$ The prevalence of lean NAFLD differs significantly due to urbanisation and wealth of different regions of the world, as well as the associated diet and physical activity levels. ${ }^{8}$

\section{Visceral adiposity}

According to Javed et $\mathrm{al}^{9}{ }^{9}$ BMI may be wrongly classified in over a quarter of children with excess adiposity. In a cohort study of 126 subjects between 11 and 17 years with similar total fat values, NAFLD patients had higher amount of visceral fat tissue. ${ }^{10}$ Owing to the importance of visceral adiposity, the least invasive and easily accessible methods are necessary. One of the proposed techniques is sagittal abdominal diameter (SAD), which is measured with a sagittometer as the distance between the calliper blades at the end of normal expiration. There is no consensus on the optimal landmark for SAD measurement. The anteroposterior diameter is measured in supine position, which allows for the displacement of subcutaneous adipose tissue laterally. Yim et $\mathrm{al}^{11}$ considered the ability of SAD to index abdominal fat more precisely than waist circumference and BMI, particularly among young and lean patients. Compared with other anthropometric measurements such as waist circumference, waist-to-hip ratio, and BMI, SAD showed stronger correlations with metabolic syndrome risk factors. ${ }^{12}$

\section{Genetic and environmental factors}

Genetic factors associated with increased risk of developing NAFLD in non-obese patients include nucleotide polymorphisms in patatin-like phospholipase domain-containing 3 (PNPLA3) [rs 738409], cholesteryl ester transfer protein (CETP) [rs12447924 and rs12597002], transmembrane 6 superfamily member 2 (TM6SF2), and apolipoprotein 3 (APCO3) [rs2854116 and rs2854117].2,13 The microsomal triglyceride transfer protein $(M T P)$, membrane-bound O-acyltransferase domaincontaining protein 7 (MBOAT7), peroxisome proliferator-activated receptor- $\gamma$ coactivator (PPARGC1A), heme oxygenase-1 (HO-1), and hypoxia inducible factor 3 alpha subunit (HIF3A) genes represent NAFLD risk factors in the paediatric 
population, but to date studies have only involved obese children. ${ }^{13}$

The relationship between dietary fructose intake and NAFLD has also been described. In a study of non-obese young patients, it was found that sweetened beverage consumption was the standalone risk factor of NAFLD development. ${ }^{14}$ Another important factor is a high cholesterol diet, even if the overall energy density is not excessive. Oxysterol (a metabolite of cholesterol), which is a ligand for the liver $\mathrm{X}$ receptor and mediating sterol regulatory element-binding protein 1c activation, can initiate fatty acid synthesis and stimulate lipogenesis (Fig). ${ }^{15}$

\section{Clinical and metabolic characteristics}

There is a paucity of data on the clinical characteristics of children with lean NAFLD. Most of studies have shown that lean subjects with NAFLD are younger, male with lower BMI and waist circumference compared with obese NAFLD. ${ }^{2}$ Comparing adolescents with lean NAFLD and healthy controls, hypertriglyceridaemia, higher levels of aspartate aminotransferase and gamma-glutamyl transferase were found, but the differences in the levels of total cholesterol, low-density lipoprotein, and highdensity lipoprotein were not statistically significant. ${ }^{4}$ In a Korean study comparing obese and non-obese paediatric patients with NAFLD, triglyceride and total cholesterol levels were significantly higher in the lean NAFLD group. However, levels of alanine aminotransferase, aspartate aminotransferase, gamma-glutamyl transferase, low-density lipoprotein, high-density lipoprotein, and uric acid did not differ between groups. ${ }^{16}$ Non-obese NAFLD subjects, deprived of other metabolic risk factors, more frequently had insulin resistance. ${ }^{17}$ In the US, insulin resistance was found to be more common in lean adolescents with NAFLD than in lean healthy controls, although this difference was not statistically significant. $^{4}$

\section{Histological features}

Liver biopsy is the gold standard in diagnosing and grading the severity of NAFLD. ${ }^{1}$ There have been limited studies devoted to the evaluation of histological features in lean children with NAFLD. In one which evaluated intrahepatic iron in patients with NAFLD, no histological differences in liver damage were found among paediatric patients with NAFLD with normal weight, overweight, or obesity. ${ }^{18}$

\section{Screening}

Population screening for NAFLD is not recommended in children but should be considered in high-risk populations. ${ }^{1}$ Because NAFLD is often an incidental finding, especially in patients with normal body weight, anthropometric measures should be a routine procedure in examination protocol in search of abdominal obesity. The diagnostic approach for patient with NAFLD is as follows. The first stage is

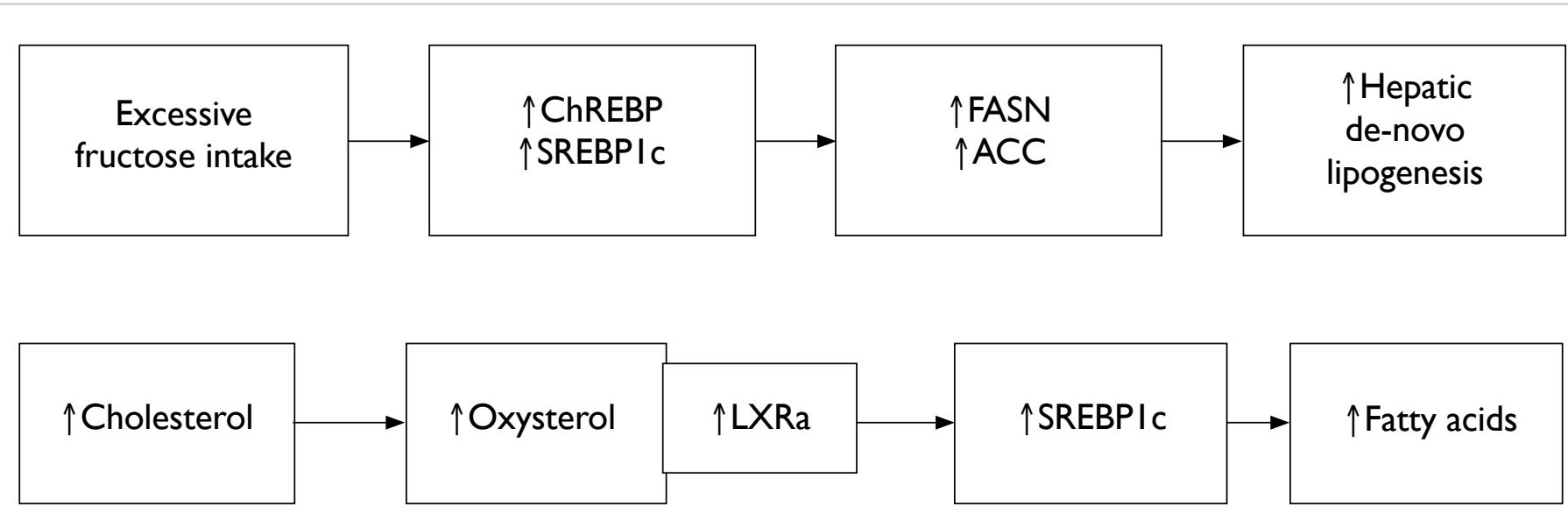

FIG. Schema of cholesterol metabolism in hepatocytes and effect of fructose intake on hepatic lipogenesis. Excessive consumption of fructose leads to activation of transcription factors (eg, ChREBP and SREBPIc) and enzymes regulating lipid synthesis (eg, FASN and ACC). High cholesterol intake causes an increase in liver cholesterol. The cholesterol-derived oxysterol ligand for LXRa mediates the activation of SREBPIc, which initiates the synthesis of fatty acids in the liver

Abbreviations: $A C C=$ acetyl-CoA carboxylase; ChREBP = carbohydrate-responsive element-binding protein; FASN = fatty acid synthase; $L X$ Ra $=$ liver $X$ receptor; SREBPIc = sterol regulatory element-binding protein Ic 
the evaluation of alanine aminotransferase activity (comparable with obese patients). The aetiology of elevated serum alanine aminotransferase is usually established through an insightful assessment of medical history, a series of screening blood tests for causes of chronic liver disease. Owing to the limited amount of data on the use of supportive imaging studies to assess hepatic steatosis in lean children, no recommendations have been made. ${ }^{2}$

\section{Treatment}

To date, there are no guidelines for treating non-obese patients with NAFLD. Decreased consumption of fructose-sweetened food and soft drinks may be appropriate. ${ }^{2}$ Increased physical exercises, especially aerobic exercise, can reduce hepatic lipid concentration and visceral adipose tissue. ${ }^{19}$ At present, pharmacological therapy for NAFLD is directed at improving the components of metabolic syndrome. In cases of lean NAFLD, one of the therapeutic options seems to be liraglutide, a glucagon-like peptide-1 analogue. In a randomised placebo-controlled study, liraglutide led to histological resolution of non-alcoholic steatohepatitis in adults with lean NAFLD. ${ }^{20}$ In 2019 the US Food and Drug Administration approved liraglutide for treatment of paediatric patients aged $\geq 10$ years with type 2 diabetes. Studies of evaluating glucagon-like peptide-1 analogues in paediatric patients with NAFLD was not conducted.

\section{Conclusion}

No data are currently available on the natural history and prognosis for lean paediatric NAFLD patients. Owing to their correct body mass, hepatic steatosis is often under-recognised. Only lifestyle interventions are recommended, including a comprehensive approach to weight management, healthy diet, and exercise.

\section{Author contributions}

All authors contributed to the concept of the study, acquisition and analysis of the data, drafting of the manuscript, and critical revision of the manuscript for important intellectual content. All authors had full access to the data, contributed to the study, approved the final version for publication, and take responsibility for its accuracy and integrity.

\section{Conflicts of interest}

All authors have disclosed no conflicts of interest.

\section{Funding/support}

This commentary received no specific grant from any funding agency in the public, commercial, or not-for-profit sectors.

\section{References}

1. Vos MB, Abrams SH, Barlow SE, et al. NASPGHAN clinical practice guideline for the diagnosis and treatment of nonalcoholic fatty liver disease in children: recommendations from the Expert Committee on NAFLD (ECON) and the North American Society of Pediatric Gastroenterology, Hepatology and Nutrition (NASPGHAN). J Pediatr Gastroenterol Nutr 2017;64:31934.

2. Wang AY, Dhaliwal J, Mouzaki M. Lean non-alcoholic fatty liver disease. Clin Nutr 2019;38:975-81.

3. Wesolowski SR, Kasmi KC, Jonscher KR, Friedman JE. Developmental origins of NAFLD: a womb with a clue. Nat Rev Gastroenterol Hepatol 2017;14:81-96.

4. Conjeevaram Selvakumar PK, Kabbany MN, Lopez R, Rayas MS, Lynch JL, Alkhouri N. Prevalence of suspected nonalcoholic fatty liver disease in lean adolescents in the United States. J Pediatr Gastroenterol Nutr 2018;67:759.

5. Fu CC, Chen MC, Li YM, Liu TT, Wang LY. The risk factors for ultrasound-diagnosed non-alcoholic fatty liver disease among adolescents. Ann Acad Med Singapore 2009;38:157.

6. WHO Expert Consultation. Appropriate body-mass index for Asian populations and its implications for policy and intervention strategies. Lancet 2004;363:157-63.

7. Alberti KG, Zimmet PZ. Definition, diagnosis and classification of diabetes mellitus and its complications. Part 1: Diagnosis and classification of diabetes mellitus provisional report of a WHO consultation. Diabet Med 1998;15:539-53.

8. Liu CJ. Prevalence and risk factors for non-alcoholic fatty liver disease in Asian people who are not obese. J Gastroenterol Hepatol 2012;27:1555-60.

9. Javed A, Jumean M, Murad MH, et al. Diagnostic performance of body mass index to identify obesity as defined by body adiposity in children and adolescents: a systematic review and meta-analysis. Pediatr Obes 2015;10:234-44.

10. Ramírez-Vélez R, Izquierdo M, Correa-Bautista JE, et al. Liver fat content and body fat distribution in youths with excess adiposity. J Clin Med 2018;7:528.

11. Yim JY, Kim D, Lim SH, et al. Sagittal abdominal diameter is a strong anthropometric measure of visceral adipose tissue in the Asian general population. Diabetes Care 2010;33:2665-70.

12. Ohrvall M, Berglund L, Vessby B. Sagittal abdominal diameter compared with other anthropometric measurements in relation to cardiovascular risk. Int J Obes Relat Metab Disord 2000;24:497-501.

13. Goyal NP, Schwimmer JB. The genetics of pediatric nonalcoholic fatty liver disease. Clin Liver Dis 2018;22:5971.

14. Assy N, Nasser G, Kamayse I, et al. Soft drink consumption linked with fatty liver in the absence of traditional risk factors. Can J Gastroenterol 2008;22:811-6.

15. Enjoji M, Yasutake K, Kohjima M, Nakamuta M. Nutrition and nonalcoholic fatty liver disease: the significance of cholesterol. Int J Hepatol 2012;2012:925807.

16. Kim EJ, Kim HJ. Nonalcoholic fatty liver disease in obese and nonobese pediatric patients. Korean J Pediatr 2019;62:30-5.

17. Bugianesi E, Gastaldelli A, Vanni E, et al. Insulin resistance in non-diabetic patients with non-alcoholic fatty liver disease: sites and mechanisms. Diabetologia 2005;48:634- 
42.

18. Manco M, Alisi A, Real JF, et al. Early interplay of intrahepatic iron and insulin resistance in children with nonalcoholic fatty liver disease. J Hepatol 2011;55:647-53.

19. Johnson NA, Sachinwalla T, Walton DW, et al. Aerobic exercise training reduces hepatic and visceral lipids in obese individuals without weight loss. Hepatology 2009;50:1105-12.

20. Armstrong MJ, Gaunt P, Aithal GP, et al. Liraglutide safety and efficacy in patients with non-alcoholic steatohepatitis (LEAN): a multicentre, double-blind, randomised, placebocontrolled phase 2 study. Lancet 2016;387:679-90. 